\title{
ON THE NONIDENTITY OF WEAK AND STRONG EXTENSIONS OF DIFFERENTIAL OPERATORS ${ }^{1}$
}

\author{
ROBERT D. MOYER
}

Since the celebrated theorem of Friedrichs [2], various authors have studied the question of the identity of weak and strong extension of partial differential operators (e.g., see [1], [3], [4], [5], [8] and [6]). In the last work, Phillips and Sarason have shown that their versions of weak and strong extensions need not agree. Recently, Ralston [7] has modified this example to show that the same is true for the usual weak and strong extensions. Here we would like to indicate another example whose basic feature is that the boundary matrix has constant nonzero eigenvalues.

The operator is the Cauchy-Riemann operator

$$
L=\left(\begin{array}{rr}
-\partial / \partial x & \partial / \partial y \\
\partial / \partial y & \partial / \partial x
\end{array}\right)
$$

on the unit square $\Omega: 0<x<1,0<y<1$. The boundary matrix is readily seen to be

$$
\pm\left(\begin{array}{rr}
-1 & 0 \\
0 & 1
\end{array}\right) \text { and } \pm\left(\begin{array}{ll}
0 & 1 \\
1 & 0
\end{array}\right)
$$

on the segments $x=1, x=0, y=1$, and $y=0$ respectively of the boundary $\partial \Omega$. For the boundary conditions we choose the self-adjoint conditions

$$
\begin{aligned}
& u_{1}=-u_{2} \text { on } x=0 \text { and } x=1 \text {, } \\
& u_{2}=0 \quad \text { on } y=0 \text { and } y=1 \text {. }
\end{aligned}
$$

With these conditions, observe that $i L$ is formally self-adjoint.

Now, using polar coordinates, set $v=r^{-1 / 2}(\cos \theta / 2,-\sin \theta / 2)$. Since the components of $v$ are the real and imaginary parts of the analytic function $z^{-1 / 2}$, it follows that $L v=0$ on $\Omega$. Also note that $v \in \mathfrak{L}_{2}(\Omega)$, and that $v$ satisfies (1) on $x=0$ and on $y=0$. Next, let $\phi$ be a smooth scalar function of $r$ such that $\phi=1$ near $r=0$ and $\phi=0$ near $r=1$. Then the function $u=\phi v$ satisfies (1) on all of $\partial \Omega$. Setting $f=L u$, one finds that $f=(L \phi) v=\phi^{\prime}(r) r^{-1 / 2}(-\cos \theta / 2, \sin \theta / 2)$. We claim that $L u=f$ weakly but not strongly (in the sense of Friedrichs).

Received by the editors January 25, 1967.

${ }^{1}$ Prepared under the auspices of NSF Grant GP-5421. 
That $L u=f$ weakly follows from the usual application of the divergence theorem together with the fact that $u=O\left(r^{-1 / 2}\right)$ as $r \rightarrow 0$. If $L u=f$ strongly, the formal self-adjointness of $i L$ would imply that

However,

$$
\int_{\Omega} f \cdot u d x d y=\int_{\Omega} L u \cdot u d x d y=0
$$

$$
\int_{\Omega} f \cdot u d x d y=-\int_{0}^{1} d r \int_{0}^{\pi / 2} r d \theta \phi(r) \phi^{\prime}(r) r^{-1}=\pi / 4 .
$$

Therefore, $u$ is not a strong solution of $L u=f$.

On the other hand, Sarason [8] has shown that his versions of the weak and strong extensions of $L$ do agree. This example fails there since Sarason require $\mathfrak{L}_{2}$ boundary values.

As a sidelight, observe that $i L$ is a formally self-adjoint operator whose closure is not a self-adjoint operator in $\mathfrak{L}_{2}(\Omega)$. Also note that (2) implies that the closure of $-L$ is not a maximal dissipative operator in $\mathcal{L}_{2}(\Omega)$, although the boundary conditions are maximal nonpositive with respect to the boundary matrix.

Finally, we note that the same example is valid for the domain $\Omega: 0<x<1,-1<y<1$, where the conditions are now

$$
\begin{array}{ll}
u_{1}=-u_{2} & \text { on } \quad x=0, \quad 0<y<1, \\
u_{1}=u_{2} & \text { on } \quad x=0,-1<y<0
\end{array}
$$

and are suitably chosen on the remainder of $\partial \Omega$. This suggests that the difficulty arises from a discontinuity in the boundary conditions.

\section{REFERENCES}

1. H. O. Cordes, Boundary problems regardless of type, Lecture Notes, Univ. of California, Berkeley, 1960.

2. K. O. Friedrichs, The identity of weak and strong extensions of differential operators, Trans. Amer. Math. Soc. 55 (1944), 132-151.

3. - Symmetric positive linear differential equations, Comm. Pure Appl. Math. 11 (1958), 333-418.

4. L. Hörmander, Weak and strong extensions of differential operators, Comm. Pure Appl. Math. 14 (1961), 371-379.

5. P. D. Lax and R. S. Phillips, Local boundary conditions for dissipative symmetric linear differential operators, Comm. Pure Appl. Math. 13 (1960), 427-455.

6. R. S. Phillips and L. Sarason, Singular symmetric positive first order differential operators, J. Math. Mech. 15 (1966), 235-272.

7. J. V. Ralston, Symmetric positive differential operators for which "weak $\neq$ strong," (unpublished).

8. L. Sarason, On weak and strong solutions of boundary value problems, Comm. Pure Appl. Math. 15 (1962), 237-288.

Pennsyluania State University 